\title{
Design and ergonomics of package inserts of drugs in Brazil: a reality in construction
}

\author{
Charles Ricardo Leite da Silva ${ }^{\mathrm{a},{ }^{*}}$ and Marcelo Marcio Soares ${ }^{\mathrm{b}, \uparrow}$

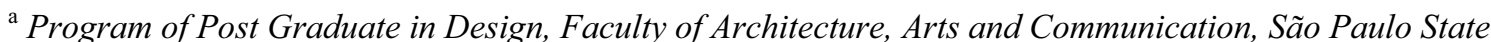 \\ University, Av. Eng. Luiz E. C. Coube, 14-01, Bauru, São Paulo, Brazil \\ ${ }^{\mathrm{b}}$ Program of Post Graduate in Design, Arts and Communication Center, Federal University of Pernambuco, Av. \\ Prof. Moraes Rego, 1235 - Cidade Universitária, Recife, Pernambuco, Brazil
}

\begin{abstract}
This research deals with the design of leaflets of medicines, evidencing the problems resulting from the lack of Brazilian normalization to promote the use of the graphical representation of instructional texts warnings. It approaches studies related to the effectiveness and efficiency of information systems, highlighting the semiotics and the cultural and informational ergonomics. The analysis of the context uses as method, an analytical study on selected warnings of thirty leaflets of medicines, followed by interviews lead with the public managers involved with the regulation of the pharmaceutical companies, and two experiments with users performed in city of Recife, in State of Pernambuco: one aiming at to identify how they interact with the leaflets of medicines, and the second one testing their understanding concerning standardized illustrations in the United States and the South Africa. The results show the need for improvements in presentation and graphic representation of leaflets of medicines, powering them to the role of communication, to ensure the consumption of medicine safely by its users. The conclusion congregates parameters and recommendations for the graphic representation of warnings in leaflets of medicines in Brazil.
\end{abstract}

Keywords: medicines, ergonomics, visual representation, cultural ergonomics, public health

\section{Introduction}

This paper discusses the design of package inserts of drugs, aiming to report on research comprehensibility of graphical representations of these warnings in package inserts in different socio-cultural realities of the country and the absence of Brazilian standards to promote the use of graphical representations of these warnings instructional texts.

The semiotic approach was highlighted in this research, since the design [1]. It's a discipline that produces not only material realities but fills communicative functions. So make sure that the drugs do not only refer to health care, but loaded with symbols, are objects of culture.
Cultural diversity of countries with large dimensions, such as Brazil, may interfere with health care, considering that drugs are present in most actions to combat diseases and ailments. Ergonomics is the branch of cultural ergonomics approach, which considers situations and characteristics, based on cultural diversity [2].

In this context, the informational ergonomics is a discipline that cuts across many subjects, including those related to perception, as the use of color, clarity, readability, comprehensibility, among other topics. We observe that it involves the analysis and information design for an effective use by users, resulting in your satisfaction and respect their diversity in terms of abilities and limitations.

\footnotetext{
* Corresponding authors e-mail: charles.leite@gmail.com

† marcelo2@nlink.com.br
} 


\section{Design and human factors of leaflets of medicines}

Instructions, cautions and warnings accompanying consumer products and or are inserted in them. Can be found in packaging and package inserts of drugs or even cutting-edge technological products, such as equipment or computer programs, and are often also found in educational materials (instructional) or health care instructions.

With the increased heating of commercial relations in the international market, there was an increase in importance of instructional information and warnings that can be disseminated and communicated in order to reach an ever larger and more heterogeneous pub- lic. There is, however, the need to overcome barriers and cultural and language differences of consumers.

In order to be granted the full use of this information by users and which is preserved the safety of such users.

According to the political on drugs Brazilian [3], governmental actions, and address the adverse effects will be used to ensure the rational use of medicines. For this purpose, should be developed and encouraged studies on the use of products as needed to contribute to the rational use of medicines.

In some countries, there is the use of standard American pharmaceutical pictograms developed by the USP Drug Information (USPDI), like figure 1.
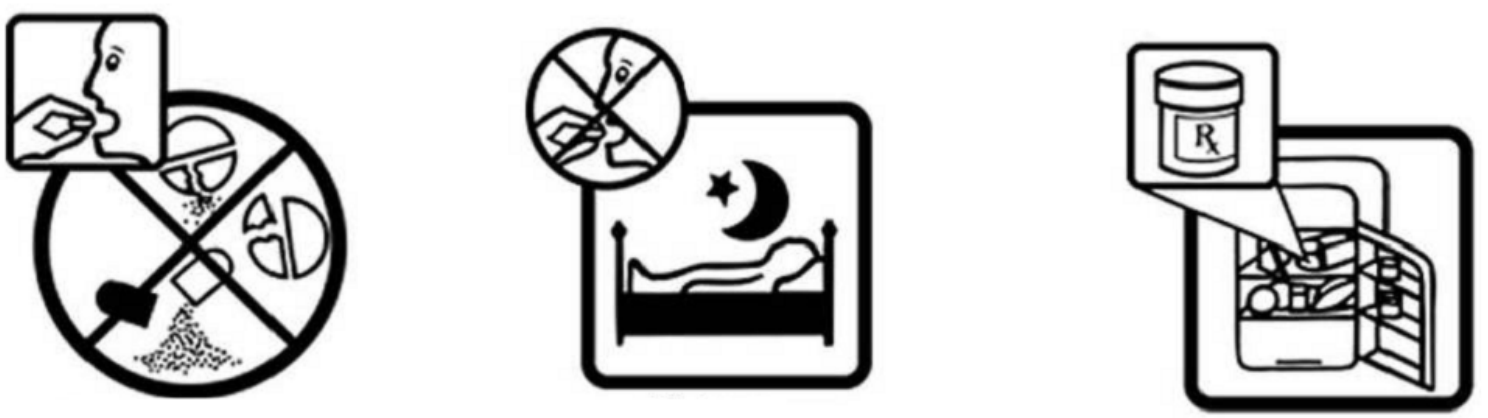

Figure 1: Pictograms of warnings on drugs of USP-DI [4]

The composition of package inserts of drugs routinely takes place in a simple manner. However, despite various devices in the printing industry and the precepts of secular printing technique. It is observed that many of these artifacts do not meet what we are offered. That is, the package insert that accompanies the medication does not help people effectively drug users.

Understanding of instructions is essential to human health and effectiveness of medicines. In some cases, the low level of education is a barrier to the understanding of health information in written form [5].

In this case, the inclusion of graphics or visual elements like images, colors and shapes, can facilitate understanding of this information.

\section{Methods}

To meet the proposed objectives, the methodology was divided into three phases:

First, we call context evaluation, we identified and we know the characteristics, goals and processes of elaboration of the warnings on medicines.

Later, there were conducted two experimental studies with potential users. An investigating how users interpret the information leaflets. The other tested the responsiveness of drug warnings. Next, we compared the findings to the results of research on perceived with citizens in South Africa[5].

The reading of texts is not nonlinear guided by the textual syntagmas, but for pragmatic guidelines [6]. It is up to the reader to establish the sequence of a connection between the elements. In the case of nonlinear texts, texts they are built according to certain logic paradigm, the logic elements located in the cen- 
ter and the margin or on the left and right, for example, leaving the reader to connect them, like figure 2 , in sequence.
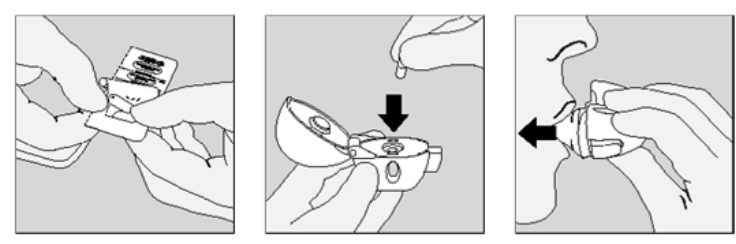

Figure 2: Visual instructions on the leaflets of medicines.

Finally, gathering the information found, this was the proposal of recommendations for the design of leaflets in Brazil, based on the results in moments earlier.

\section{Results and discussion}

The context evaluation showed that there are inserts for medicines that do not display simple information such as adverse reactions and drug interactions. It was observed that in some warnings indicate the danger, but does not show the possible negative consequences. Also, we identified that the main drug involved in accidents are children and elderly. As, in which Brazilian health system, trying to centralize the management of information on medicines, leaving local managers uninformed, which makes an effective control of incidents and accidents of consumption.

The first experimental study mapped and proved to be too low to complete identification (only 10\% of the sample) of the warnings in package inserts of drugs. The second experimental study, which tested the comprehensibility of pictorial warnings, demonstrated its use to generate timely input into the development of illustrations in the inserts.

Regarding the parameters for the development of new labels of medicine, especially in Brazil, were grouped as follows: (i) the informational content, indicating the expressions and terms that must be present, (ii) for presentation and representation graphics, recommending leaflets printed in color favoring the highlight of the information hierarchy, allowing relevant information, (iii) the management information system of medicines, we recommend the use of information technology to improve the system for reporting complaints and events adverse by users, (iv) in terms of culture, one must select common words in use throughout the country and, where possible, illustrations to anchor what has been said verbally, and (v) regarding the accessibility of information, it is suggested the inclusion of warnings on the external packaging of drugs.

Assessment context, the results indicate that, in general, is highly diversified in form or manner as are the warnings in package inserts from different forms of delimitation of the area, employing various emphatic elements (colour, contrast, use of arrows and bullets) and reduced use of symbolic elements (illustrations). Also, it was noted from interviews with public officials implicated in the regulation of pharmaceutical companies in Brazil, there are flaws in the reporting system of incidents and accidents involving medications.

In turn, detects that this type of performance evaluation conducted by designers collaborate with improvements in the design process because it provides the necessary feedback to them.

\section{Conclusion}

While it is not possible to eliminate elements of danger or access by people using some products, in inclusion of warnings is presented as a strategy to minimize risks of misuse of product. In this case, it would give the warnings necessary subsidy minimization of risks to health and safety of consumer. The natural toxicity of medications that are useful for people should be used for a specified period, in specified amount, when necessary, prevent the removal of risk inherent to uses

It was perceived that the pharmaceutical industry in Brazil isn't effectively regulated.

Thus, it is clear that many companies don't care about the quality of information passed on to consumers, presenting information sometimes totally ineffective.

We identified the gap among research usability of information systems focused on drugs. Since, they don't consider as stakeholders, those involved in the regulatory process.

Many studies, only consolidate a satisfaction survey with users.

Finally, remember that the design should always meet the demands of society. So, that kind of research can contribute much to improved public health in Brazil and elsewhere. 
For other studies on the subject of this research in the area, with objectives similar to those proposed here, suggests that some recommendations are committed to improving people's research and contribute to a better success of achievements, as increasing the number of drug package inserts examined, to take a more representative sample of the large number of drugs marketed in Brazil. The choice of specific number of those who could be in the results become statistically significant for researches.

\section{References}

[1] B.E. Bürdek. Design: history, theory and practice of products design. São Paulo: Blucher, 2006.

[2] T.L. Smith-Jackson; M.S. Wogalter Applying cultural ergonomics - human factors to safety information research. In: Proceedings of 7 th conference of Union of Latin-American Ergonomics. 2002.

[3] BRASIL. Ministério da Saúde. Secretaria de Políticas de Saúde. Departamento de Atenção Básica. Política Nacional de Medicamentos. Brasília: Ministério da Saúde, 2001.

[4] USP - The United States Pharmacopeial Convention Inc 1998. available in: www.usp.org/audiences/consumers/pictograms Accessed: 15 jan. 2007
[5] R. Dowse; M. Ehlers. Pictograms for conveying medicine instructions: comprehension in various South African language groups. In: South African Journal of Science, 100, Nov/Dec. 2004. pp. 687-693.

[6] J. L Lemke. Travels in hypermodality. v1. London, Thousand Oaks, CA, New Delhi: SAGE Publications, 2002. pp. 299-325.

\section{Special thanks}

The National Council for Scientific and Technological Development (CNPq), agency linked to the Ministry of Science and Technology of Brazil, for providing financial support for research reported. 